TRANSACTIONS OF THE

AMERICAN MATHEMATICAL SOCIETY

Volume 358, Number 7, Pages 3113-3131

S 0002-9947(06)03815-3

Article electronically published on March 1, 2006

\title{
COMMUTATIVE IDEAL THEORY WITHOUT FINITENESS CONDITIONS: COMPLETELY IRREDUCIBLE IDEALS
}

\author{
LASZLO FUCHS, WILLIAM HEINZER, AND BRUCE OLBERDING
}

\begin{abstract}
An ideal of a ring is completely irreducible if it is not the intersection of any set of proper overideals. We investigate the structure of completely irrreducible ideals in a commutative ring without finiteness conditions. It is known that every ideal of a ring is an intersection of completely irreducible ideals. We characterize in several ways those ideals that admit a representation as an irredundant intersection of completely irreducible ideals, and we study the question of uniqueness of such representations. We characterize those commutative rings in which every ideal is an irredundant intersection of completely irreducible ideals.
\end{abstract}

\section{INTRODUCTION}

Let $R$ denote throughout a commutative ring with 1 . An ideal of $R$ is called irreducible if it is not the intersection of two proper overideals; it is called completely irreducible if it is not the intersection of any set of proper overideals. Our goal in this paper is to examine the structure of completely irreducible ideals of a commutative ring on which there are imposed no finiteness conditions. Other recent papers that address the structure and ideal theory of rings without finiteness conditions include [3], 4], 8], 10], 14, [15], 16], 19], 25], 26].

A proper ideal $A$ of $R$ is completely irreducible if and only if there is an element $x \in R$ such that $A$ is maximal with respect to not containing $x$. Indeed, the condition is clearly sufficient for $A$ to be completely irreducible since $x$ is in the intersection of the proper overideals of $A$. On the other hand, if $A$ is completely irreducible and $x$ is an element that is not in $A$ but is in the intersection of the proper overideals of $A$, then $A$ is maximal with respect to not containing $x$. Evidently, maximal ideals are completely irreducible. If $R$ is a domain (not a field), then the zero ideal of $R$ is prime and irreducible, but it is not completely irreducible (it is the intersection of all nonzero ideals). More generally, a prime ideal is always irreducible and is completely irreducible if and only if it is a maximal ideal. In Theorem 1.3 we characterize completely irreducible ideals in various ways. We deduce in Corollary [1.5 that an irreducible ideal of a Noetherian ring is completely irreducible if and only if it is primary for a maximal ideal.

A great deal is known about the structure of the irreducible ideals of a Noetherian ring. Indeed, the first decomposition theorem established by Emmy Noether [27]

Received by the editors December 23, 2003 and, in revised form, June 4, 2004 and July 26, 2004.

2000 Mathematics Subject Classification. Primary 13A15, 13 F05.

Key words and phrases. Irreducible ideal, completely irreducible ideal, irredundant intersection, arithmetical ring. 
states that each ideal of a Noetherian ring admits a representation as an irredundant intersection of finitely many irreducible ideals; moreover, the number of components appearing in such a representation is an invariant, and each such representation is reduced (i.e., no ideal in the representation can be replaced by a strictly larger ideal to obtain the same intersection). Another result due to Noether [27] is that a proper irreducible ideal of a Noetherian ring is a primary ideal. In a ring without finiteness assumptions there may exist proper irreducible ideals that are not primary. Fuchs in 12 introduced the concept of a primal ideal, where a proper ideal $A$ of $R$ is said to be primal if the zero-divisors in $R / A$ form an ideal. The ideal of zero-divisors is then necessarily of the form $P / A$, where $P$ is a prime ideal of $R$ called the adjoint prime of $A$. We also say that $A$ is $P$-primal. In Fuchs [12] it is shown that proper irreducible ideals are primal. More can be said about completely irreducible ideals. We observe in Theorem 1.3 several equivalences to a proper ideal $C$ of $R$ being completely irreducible, among which is that $C$ is irreducible and $R / C$ contains a simple submodule.

A ring in which every irreducible ideal is completely irreducible is zero dimensional. Corollary 1.5 states that if $\operatorname{dim} R=0$ and each primary ideal of $R$ contains a power of its radical, then every irreducible ideal of $R$ is completely irreducible. In Section 2 we address the question: Under what conditions is an ideal representable as an irredundant intersection of completely irreducible ideals? We consider in Section 3 the question of uniqueness of representation of the ideal $A$ as an irredundant intersection of completely irreducible ideals. In Section 4 we characterize the rings in which every ideal is an irredundant intersection of completely irreducible ideals. We prove in Theorem 4.2 that every ideal of the ring $R$ is an irredundant intersection of completely irreducible ideals exactly if the ring is semi-artinian, where a ring $R$ is said to be semi-artinian if every nonzero $R$-module contains a simple $R$-module.

Proposition 1.4 and Corollary 1.5 characterize the completely irreducible ideals of a Noetherian ring. In Section 5 we give an explicit description of the completely irreducible ideals of an arithmetical ring, where the $\operatorname{ring} R$ is arithmetical if for every maximal ideal $M$ the ideals of the localization $R_{M}$ are linearly ordered with respect to inclusion. An arithmetical integral domain is a Prüfer domain. For a prime ideal $P$ of a Prüfer domain, Fuchs and Mosteig prove in [17, Lemma 4.3] that the $P$-primal ideals form a semigroup under ideal multiplication. We generalize this result in Theorem 5.6 For a prime ideal $P$ of an arithmetical ring, we show that the regular $P$-primal ideals form a semigroup under ideal multiplication, where an ideal is regular if it contains a nonzerodivisor. Theorem 5.8 states that if $M$ is a maximal ideal of an arithmetical ring $R$, then the set $\mathcal{F}$ of completely irreducible regular ideals of $R$ with adjoint maximal ideal $M$ is closed under ideal-theoretic multiplication, and $\mathcal{F}$ with this multiplication is a totally ordered cancellative semigroup.

A good reference for our terminology and notation is [18. For ideals $I, J$ of the ring $R$, the residual $I: J$ is defined as usual by

$$
I: J=\{x \in R: x J \subseteq I\} .
$$

For an ideal $A$ and for a prime ideal $P$ of $R$, we use the notation

$$
A_{(P)}=\{x \in R: s x \in A \text { for some } s \in R \backslash P\}=\bigcup_{s \in R \backslash P} A: s
$$


to denote the isolated $P$-component (isoliertes Komponentenideal) of $A$ in the sense of Krull [24, page 16]. Notice that $x \in A_{(P)}$ if and only if $A: x \nsubseteq P$. If $R$ is a domain, then $A_{(P)}=A R_{P} \cap R$, where $R_{P}$ denotes the localization of $R$ at $P$.

Two different concepts of associated primes of a proper ideal $A$ of the ring $R$ are useful for us. One of these was introduced by Krull [23, page 742], and following 22 we call a prime ideal $P$ of $R$ a Krull associated prime of $A$ if for every $x \in P$, there exists $y \in R$ such that $x \in A: y \subseteq P$. The prime ideal $P$ is said to be a strong Bourbaki associated prime of $A$ if $P=A: x$ for some $x \in R$.

\section{IRREducible AND COMPLETELY IRREDUCiBle idEALS}

A ring is called subdirectly irreducible if in any of its representations as a subdirect product of rings, one of the projections to a component is an isomorphism. It is straightforward to see:

Lemma 1.1. A proper ideal $C$ of $R$ is completely irreducible if and only if the factor ring $R / C$ is subdirectly irreducible, or equivalently if and only if the $R$-module $R / C$ has a simple essential socle.

Thus if $C$ is completely irreducible, then $R / C$ contains a minimal nonzero ideal $C^{*} / C$ : the intersection of all nonzero ideals of $R / C$; this is then the essential socle of $R / C$. We shall call $C^{*}$ the cover of $C$. Clearly, $C^{*} / C$ is a simple $R$-module, so $C^{*} / C \cong R / M$ for a maximal ideal $M$ of $R$. (If $C$ is only assumed to be irreducible, then we can only claim that $R / C$ is a uniform $R$-module, that is, the intersection of any two nonzero submodules of $R / C$ is not zero.)

Proposition 1.2. A completely irreducible proper ideal $C$ of the ring $R$ is a primal ideal whose adjoint prime is the maximal ideal $M$ of $R$ for which $C^{*} / C \cong R / M$. Furthermore, $M$ is a strong Bourbaki associated prime of $C$.

Proof. Let $x \in R$ be a representative of any coset of $C$ generating $C^{*} / C \cong R / M$. Then $x \notin C$ and $x M \subseteq C$. Hence $M \subseteq C: x \subset R$ (proper containment); thus we necessarily have $M=C: x$, so $M$ is a strong Bourbaki associated prime of $C$. Evidently, $M$ has to be the adjoint prime of the primal ideal $C$.

Completely irreducible ideals can be characterized in various ways as we demonstrate in Theorem 1.3 .

Theorem 1.3. For a proper ideal $C$ of $R$, the following conditions are equivalent:

(i) $C$ is completely irreducible;

(ii) the factor module $R / C$ is an essential extension of a simple module;

(iii) $C$ is an irreducible ideal and $R / C$ contains a simple $R$-submodule;

(iv) $C$ is an irreducible ideal and $C$ is properly contained in $C: M$ for some maximal ideal $M$ of $R$;

(v) $C$ is irreducible with adjoint prime a maximal ideal $M$ of $R$, and $M=C: x$ for some $x \in R \backslash C$;

(vi) $C=C_{(M)}$ for some maximal ideal $M$ of $R$ and $C R_{M}$ is a completely irreducible ideal of $R_{M}$.

Proof. The equivalence of (i) and (ii) and of (iii) and (iv) is obvious, and so is the implication (i) $\Rightarrow$ (iv). To prove (iii) $\Rightarrow$ (i), observe that a simple submodule of a uniform module is an essential socle of the module. 
By Proposition 1.2. (i) $\Rightarrow$ (v) is clear. On the other hand, condition (v) implies that $x+C$ generates a simple $R$-submodule in $R / C$. By the uniformity of $R / C$ this simple submodule is an essential socle of $R / C$, and (i) holds.

The equivalence of (v) and (vi) follows from the following two observations: (a) if $M$ is a maximal ideal containing $C$, then $(C: x) R_{M}=C R_{M}: R_{M} x$ for any $x \in R$; and (b) $C_{(M)}$ is irreducible if and only if $C R_{M}$ is an irreducible ideal of $R_{M}$ (see Remark 1.6 of [14]).

A ring is Laskerian if every ideal has a finite primary decomposition.

Proposition 1.4. If every proper ideal of the ring $R$ is an intersection of primary ideals (possibly an infinite intersection), then every completely irreducible proper ideal of $R$ is primary for a maximal ideal. In particular, if $R$ is a Laskerian ring (or a Noetherian ring), then every completely irreducible proper ideal of $R$ is primary for a maximal ideal.

Proof. Let $C$ be a completely irreducible ideal of $R$ with adjoint prime $M$ and let $x \in R$ be a representative of any coset of $C$ generating $C^{*} / C \cong R / M$. Then $x \notin C$ and $x M \subseteq C$. Since $C$ is an intersection of primary ideals, there exists a primary ideal $Q$ of $R$ such that $C \subseteq Q$ and $x \notin Q$. Since $C$ is completely irreducible, it follows that $C=Q$. Therefore $C$ is a primary ideal that is $M$-primal, so $C$ is $M$-primary.

Corollary 1.5. Let $M$ be a maximal ideal of the ring $R$ and let $C$ be an irreducible $M$-primary ideal. If $M^{n} \subseteq C$ for some positive integer $n$, then $C$ is completely irreducible. Thus if $M$ is finitely generated, then every irreducible $M$-primary ideal is completely irreducible. Therefore an irreducible proper ideal of a Noetherian ring is completely irreducible if and only if it is primary for a maximal ideal.

Proof. If $M^{n} \subseteq C$ for some positive integer $n$, then $C \subset(C: M)$. Hence by Theorem 1.3(iv), $C$ is completely irreducible. The last statement now follows from Proposition 1.4.

Remark 1.6. We are interested in describing the rings in which every irreducible ideal is completely irreducible. Since prime ideals are irreducible and a prime ideal is completely irreducible if and only if it is maximal, the condition that every irreducible ideal in $R$ is completely irreducible implies that all prime ideals of $R$ are maximal and $\operatorname{dim} R=0$. If $R$ is a reduced zero-dimensional ring, then $R_{P}$ is a field for each $P \in \operatorname{Spec} R$ and every primal ideal of $R$ is maximal. Therefore every irreducible ideal of a reduced zero-dimensional ring is completely irreducible.

Recall that a ring $R$ is semi-artinian if every nonzero $R$-module contains a simple $R$-module. If $R$ is semi-artinian, then all irreducible ideals of $R$ are completely irreducible (see also Lemma 2.4 in Dilworth-Crawley [7]). The existence of a reduced zero-dimensional ring $R$ having no principal maximal ideals shows that a ring in which all irreducible ideals are completely irreducible need not be semi-artinian. In Example 1.7 we give two specific ways to obtain an example of such a ring $R$.

Example 1.7. (1) Let $L$ denote the algebraic closure of the field $\mathbb{Q}$ of rational numbers and let $D$ be the integral closure of the ring $\mathbb{Z}$ of integers in the field $L$. Fix a prime integer $p$ and let $R=D / \sqrt{p D}$. Then $R$ is a reduced zero-dimensional ring having the property that no maximal ideal of $R$ is finitely generated. 
(2) Let $\mathbb{Q}^{\omega}$ denote the product of countably infinitely many copies of the field $\mathbb{Q}$ of rational numbers and let $I$ denote the direct sum ideal of $\mathbb{Q}^{\omega}$. Then $R=\mathbb{Q}^{\omega} / I$ is a reduced zero-dimensional ring in which no maximal ideal is finitely generated.

Remark 1.8. Let $M$ be a maximal ideal of the ring $R$. By Theorem 1.3 an irreducible $M$-primary ideal $A$ is completely irreducible if and only if $A \subset(A: M)$. If $(R, M)$ is a rank-one nondiscrete valuation domain and $A=x R$ is a principal $M$-primary ideal, then $A$ is irreducible, but not completely irreducible. (Since the value group $G$ of $R$ is a dense subset of $\mathbb{R}$, the value of $x$ is the limit of smaller elements of $G$.) On the other hand, if $\operatorname{dim} R=0$ and if each primary ideal of $R$ contains a power of its radical, then Corollary 1.5 implies that all irreducible ideals of $R$ are completely irreducible.

Let $A \subseteq C$ be ideals of the ring $R$. If $C$ is completely irreducible, we call $C$ a relevant completely irreducible divisor of $A$ if $A$ has a decomposition as the intersection of completely irreducible ideals in which $C$ is relevant (i.e. it cannot be omitted). If $A$ admits an irredundant decomposition with completely irreducible ideals, then all the ideals in this decomposition are relevant. On the other hand, a prime ideal that is not a maximal ideal has no relevant completely irreducible divisors. In Proposition 1.9 we characterize the relevant completely irreducible divisors of an ideal.

Proposition 1.9. A completely irreducible ideal $C$ containing the ideal $A$ is a relevant completely irreducible divisor of $A$ if and only if the submodule $C / A$ of $R / A$ is not essential.

Proof. First suppose $C$ is a relevant completely irreducible divisor of $A$, and $A=$ $C \cap \bigcap_{i \in I} C_{i}$ is a decomposition with completely irreducible ideals $C_{i}$, where $C$ cannot be omitted. Then there exist an element $x \in \bigcap_{i \in I} C_{i} \backslash C$ and an $r \in R$ such that $r x \in C^{*} \backslash C$. Let $u=r x$ and let $\bar{u}$ denote the image of $u$ in $R / A$. Then $\bar{u} \notin C / A$, while if $M$ denotes the maximal ideal of $R$ such that $C^{*} / C \cong R / M$, then $M u \subseteq C \cap \bigcap_{i \in I} C_{i}=A$ implies $R \bar{u}$ is a simple submodule of $R / A$. Hence the submodule $C / A$ of $R / A$ is not essential.

Conversely, assume $C / A$ is not essential in $R / A$, i.e. there exists $x \in R \backslash A$ such that $(R x+A) \cap C=A$. Write the ideal $(R x+A)$ as an intersection of completely irreducible ideals $C_{i}(i \in I)$. Then $A=C \cap \bigcap_{i \in I} C_{i}$, where $C$ is relevant.

Corollary 1.10. For every completely irreducible ideal $C$ containing the ideal $A$, the cover $C^{\star}$ satisfies: $C^{\star} / A$ is an essential submodule in $R / A$.

Proof. If $C$ is not a relevant completely irreducible divisor of $A$, then $C / A$ is an essential submodule of $R / A$, so $C^{*} / A$ is essential in this case. On the other hand, if $C$ is relevant, then for any $x \in R \backslash C$, there exists $r \in R$ such that $r x \in C^{*} \backslash C$.

Corollary 1.11. Let $A$ be a proper ideal of the ring $R$. There exists a relevant completely irreducible divisor of $A$ if and only if the socle $\operatorname{Soc}(R / A) \neq 0$.

Proof. Assume that $C$ is a relevant completely irreducible divisor of $A$. If $A=C$, then $\operatorname{Soc}(R / A)=\operatorname{Soc}(R / C)=C^{*} / C \neq 0$. If $A \subset C$, then $A=C \cap B$, where $A \subset B$. Let $b \in B \backslash A$. Then $b \notin C$, so there exists $r \in R$ such that $r b \in C^{*} \backslash C$. It follows that $M r b \subseteq C \cap B=A$, where $M$ is the adjoint prime of $C$. Therefore $(A+r b R) / A \subseteq \operatorname{Soc}(R / A)$ and $\operatorname{Soc}(R / A) \neq 0$. 
Conversely, assume that $\operatorname{Soc}(R / A) \neq 0$. Then there exists $x \in R \backslash A$ such that $x M \subseteq A$ for some maximal ideal $M$ of $R$ with $A \subseteq M$. Let $B=A+x R$. Then $B / A \cong R / M$. Let $C$ be an ideal of $R$ containing $A$ and maximal with respect to $x \notin C$. Then $C$ is completely irreducible and $A=C \cap B$. Therefore $C$ is a relevant completely irreducible divisor of $A$.

Remark 1.12. The ring $R$ of Example 1.7 is a zero-dimensional reduced ring for which $\operatorname{Soc} R=0$. Thus in this ring, the ideal (0) has no relevant completely irreducible divisors.

In Proposition 1.13, we observe a connection between relevant completely irreducible divisors of an ideal and maximal ideals that are strong Bourbaki associated primes of the ideal.

Proposition 1.13. Let $A$ be a proper ideal of the ring $R$. A maximal ideal $M$ of $R$ is a strong Bourbaki associated prime of $A$ if and only if there exists a relevant completely irreducible divisor of $A$ that is $M$-primal.

Proof. Suppose $C$ is a relevant completely irreducible divisor of $A$ that is $M$-primal. The proof of Proposition 1.9 establishes the existence of an element $u \in R \backslash A$ such that $M u \subseteq A$. Hence $M=A: u$ is a strong Bourbaki associated prime of $A$. Conversely, if $M=A: x$, then $x \notin A$. Let $C$ be an ideal of $R$ that contains $A$ and is maximal without $x$. Then $M=C: x$ and $C$ is $M$-primal. Since every ideal is an intersection of completely irreducible ideals, there exist completely irreducible ideals $C_{i}$ such that $A+R x=\cap_{i \in I} C_{i}$. Then $(A+R x) / A$ being simple implies that $A=C \cap\left(\bigcap_{i \in I} C_{i}\right)$, where $C$ is clearly a relevant component.

\section{IRREDUNDANT INTERSECTIONS}

We consider under what conditions an ideal may be represented as an irredundant intersection of completely irreducible ideals, where, as usual, irredundant means that none of the components may be omitted without changing the intersection.

If $\left\{B_{i}\right\}_{i \in I}$ is a family of $R$-modules, then by an interdirect product of this family we mean an $R$-submodule of the direct product $\prod_{i \in I} B_{i}$ that contains the direct sum $\bigoplus_{i \in I} B_{i}$. Similarly, if $\left\{R_{i}\right\}_{i \in I}$ is a family of rings, by an interdirect product of this family of rings we mean a subring of the direct product $\prod_{i \in I} R_{i}$ that contains the direct sum $\bigoplus_{i \in I} R_{i}$ and the identity of the direct product.

In Lemma2.1 and later in this section, we use the following notation. Let $\left\{C_{i}\right\}_{i \in I}$ be a family of completely irreducible ideals of the ring $R$ and let $A=\bigcap_{i \in I} C_{i}$. For each $j \in I$ let $\bar{C}_{j}=\bigcap_{i \in I, i \neq j} C_{i}$. We frequently use the following basic fact.

Lemma 2.1. Let $A=\bigcap_{i \in I} C_{i}$ be an irredundant representation of the proper ideal $A$ with completely irreducible ideals $C_{i}$.

(i) There are elements $u_{i} \in R \backslash C_{i}(i \in I)$ such that $C_{i}^{*}=C_{i}+R u_{i}$ for $i \in I$ and $u_{i} \in C_{j}$ for all $j \neq i$.

(ii) For each $i \in I$, the $R$-module $\bar{C}_{i} / A$ has an essential socle generated by $u_{i}+A$.

(iii) In the representation $A=\bigcap_{i \in I} C_{i}$, no $C_{i}$ can be replaced by a larger ideal and still have the intersection be equal to $A$.

(iv) With $u_{i}$ as in (i), let $U_{i}=\left(R u_{i}+A\right) / A$. Then $U_{i}$ is a simple $R$-module and the socle of $R / A$ is an interdirect product of the $U_{i}$. 
Proof. (i) Since the intersection of the $C_{i}(i \in I)$ is irredundant, for each $i \in I$ there exists an element $x_{i} \in \bar{C}_{i}$ such that $x_{i} \notin C_{i}$. By Lemma 1.1, $R / C_{i}$ is subdirectly irreducible, so some multiple $r x_{i} \notin C_{i}$ has the property that its image in $R / C_{i}$ is in Soc $\left(R / C_{i}\right)$. If we choose $u_{i}=r x_{i}$ for each $i \in I$, then we obtain elements with the desired properties.

(ii) From (i) it is clear that $\left\langle u_{i}+A\right\rangle$ is in the socle of $\bar{C}_{i} / A$. By way of contradiction, suppose that there is a cyclic $R$-module $\langle v+A\rangle$ contained in $\bar{C}_{i} / A$ and independent of $\left\langle u_{i}+A\right\rangle$. As $C_{i}$ is completely irreducible, there is an $r \in R$ such that $r v-u_{i} \in C_{i}$. Then $r v-u_{i} \in C_{i} \cap \bar{C}_{i}=A$, contradicting the independence of $\langle v+A\rangle$ and $\left\langle u_{i}+A\right\rangle$.

(iii) If we replace $C_{i}$ by a larger ideal, then the intersection will contain $u_{i}$, so it will no longer represent $A$.

(iv) As $M_{i} u_{i} \subseteq A$ for a maximal ideal $M_{i}$, it is clear that the $U_{i}=\left\langle u_{i}+A\right\rangle$ are simple submodules of $R / A$. From (i) it follows that they are independent, so Soc $(R / A)$ contains their direct sum $D=\bigoplus_{i \in I} U_{i}$. Suppose there is a $v \in R$ such that $v+A \in \operatorname{Soc}(R / A)$, but not in $D$, say $M v \subseteq A$ for a maximal ideal $M$ of $R$. Thus $v \notin C_{j}$ for some $j \in I$. If $v \notin C_{j}+R u_{j}$, then $M v \subseteq C_{j}$ implies $C_{j}=$ $\left(C_{j}+R u_{j}\right) \cap\left(C_{j}+R v\right)$, contradicting the irreducibility of $C_{j}$. Hence $v \in C_{j}+R u_{j}$ for all $j$ with $v \notin C_{j}$. This means that the canonical injection $R / A \rightarrow \prod_{j \in I} R / C_{j}$ maps $v$ to an element of $\prod_{j \in I}\left(R u_{j}+C_{j}\right) / C_{j} \cong \prod_{j \in I} U_{j}$.

We remark that part (iii) of Lemma 2.1 is also a consequence of the stronger statement: if $A=B \cap C$, where $C$ is irreducible and relevant for the decomposition of $A$, then $C$ cannot be replaced by any proper overideal (see Noether [27, Hilfssatz II]). In fact, if $C$ is properly contained in $C^{\prime}$, then $A=B \cap C^{\prime}$ would lead to $C=C+\left(B \cap C^{\prime}\right)=(C+B) \cap C^{\prime}$, contradicting the irreducibility of $C$.

Remark 2.2. It will be useful to keep in mind that (iv) of Lemma 2.1 implies that

$$
\bigoplus_{i \in I} \operatorname{Soc}\left(\bar{C}_{i} / A\right) \subseteq \operatorname{Soc}(R / A) \subseteq \prod_{i \in I} \operatorname{Soc}\left(\bar{C}_{i} / A\right) .
$$

Notice also that $\operatorname{Soc}\left(\bar{C}_{i} / A\right) \cong \operatorname{Soc}\left(C_{i}+\bar{C}_{i}\right) / C_{i}=\operatorname{Soc}\left(R / C_{i}\right)=C_{i}^{*} / C_{i}$.

Next we exhibit an example where $\operatorname{Soc}(R / A)$ is the direct product of the $\operatorname{Soc}\left(\bar{C}_{i} / A\right)$.

Example 2.3. For each nonnegative integer $n$, let $Z_{n}=\left\langle x_{n}\right\rangle$ be a cyclic group of order $p$, where $p$ is a fixed prime integer. Consider the product $\prod_{n<\omega} Z_{n}$ with trivial multiplication and let $R$ be the ring obtained by adjoining an identity to $\prod_{n<\omega} Z_{n}$ in such a way that as an additive group $R=\mathbb{Z} \oplus \prod_{n<\omega} Z_{n}$ and multiplication is defined as $(a, b) \times(c, d)=(a c, a d+c b)$, where $a, c \in \mathbb{Z}$ and $b, d \in \prod_{n<\omega} Z_{n}$.

For each nonnegative integer $m$, let $C_{m} \cong \prod_{n<\omega, n \neq m} Z_{n}$ denote the subgroup of $\prod_{n<\omega} Z_{n}$ of elements having zero coordinate in component $Z_{m}$. Then $C_{m}$ is an ideal of $R$ that is maximal with respect to not containing the element that is $x_{m}$ in component $Z_{m}$ and zero in all other components. Thus $C_{m}$ is completely irreducible. We have $\bigcap_{n<\omega} C_{n}=0$ is an irredundant intersection, and in this example, the first containment relation in (1) is strict, while the second becomes equality. 
For an irredundant intersection $A=\bigcap_{i \in I} C_{i}$ with completely irreducible ideals $C_{i}$, we demonstrate in Example 2.4 that Soc $(R / A)$ may fail to be an essential submodule of $R / A$.

Example 2.4. There is a ring $R$ in which the zero ideal is an irredundant intersection of completely irreducible ideals and Soc $R$ is not an essential submodule of $R$. Let $\left\{p_{n} \mid n<\omega\right\}$ be the set of prime numbers, and let $Z_{n}$ denote the ring $\mathbb{Z} / p_{n}^{2} \mathbb{Z}(n<\omega)$. In the direct product $P=\prod_{n<\omega} Z_{n}$ consider the subring $R$ generated by $1=(1,1, \ldots, 1, \ldots), e=\left(p_{0}, p_{1}, \ldots, p_{n}, \ldots\right)$, and $r_{n}=\left(0, \ldots, 0, p_{n}, 0, \ldots\right)$ for each $n<\omega$. Then $e^{2}=0, e r_{n}=0$ and $r_{n} r_{m}=0$ for all $n, m<\omega$. Consequently, a typical element of $R$ has the form $a=m \cdot 1+s e+n_{0} r_{0}+\cdots+n_{k} r_{k}$ for some $k \geq 0$, where $m, s, n_{i} \in \mathbb{Z}$. The ideal $R e$ is just the infinite cyclic subgroup generated by $e$. Define $C_{n}$ as an ideal containing all $r_{i}(i<\omega, i \neq n), r_{n}-p_{0}^{n} p_{1}^{n} \cdots p_{n}^{n} e$, and being maximal with respect to $r_{n} \notin C_{n}$. Clearly, the intersection $\bigcap_{n<\omega} C_{n}$ contains no nonzero element of the socle $\operatorname{Soc} R=\bigoplus_{n<\omega} R r_{n}$, and is evidently irredundant. To see that it cannot contain any element outside the socle either, assume by way of contradiction that $a$ as above is contained in every $C_{n}$. Then either a multiple of $s e$ or $a e=m e$ is contained in every $C_{n}$, which is impossible, since $p_{0}^{n} p_{1}^{n} \cdots p_{n}^{n} e \notin C_{n}$. However, the socle of $R$ is not essential in $R$, as the ideal $R e$ intersects $\operatorname{Soc} R$ trivially.

Let $A$ be a proper ideal of the ring $R$. In Theorem 2.5 we relate representations of $A$ as an intersection of bigger ideals with representations of $R / A$ as a subdirect product.

Theorem 2.5. For a proper ideal $A$ of the ring $R$ the following are equivalent:

(i) $A$ is the irredundant intersection of completely irreducible ideals;

(ii) $R / A$ is an irredundant subdirect product of subdirectly irreducible $R$-algebras $R_{i}$.

Proof. (i) $\Rightarrow$ (ii) Let $\left\{C_{i}\right\}_{i \in I}$ be a family of completely irreducible ideals of $R$ such that $A=\bigcap_{i \in I} C_{i}$ is an irredundant intersection. Then $R / A$ can be identified via the canonical map $\phi: R \rightarrow \prod_{i \in I} R / C_{i}$ with the subring $\phi(R)$ of the direct product $\prod_{i \in I} R / C_{i}$, where each component $R / C_{i}$ contains a simple $R$-module $C_{i}^{*} / C_{i}$ as an essential socle, so each component is subdirectly irreducible. If we drop a component $R / C_{j}$, then $\phi$ will no longer induce an embedding of $R / A$ in the product $\prod_{i \in I, i \neq j} R / C_{i}$, so the subdirect representation is irredundant.

(ii) $\Rightarrow$ (i) Assume that $R / A$ is an irredundant subdirect product of the subdirectly irreducible $R$-algebras $R_{i}(i \in I)$. Let $T=\prod_{i \in I} R_{i}$, and let $\pi_{i}: R / A \rightarrow R_{i}$ be the $i$ th canonical projection. The kernel of $\pi_{i}$ is $C_{i} / A$, where $C_{i}$ is a completely irreducible ideal of $R$ properly containing $A$, and $R_{i}$ is isomorphic to $R / C_{i}$. Clearly, $A=\bigcap_{i \in I} C_{i}$ and this representation is irredundant.

Example 2.6. Let $F_{p}$ denote the prime field of characteristic $p$, for a prime $p$. Define $R$ as the direct product $\prod_{p} F_{p}$ with $p$ running over an infinite set of primes. Identify the product $C_{q}$ of the $F_{p}$ with $p \neq q$ ( $q$ a fixed prime) as a subset of $R$ consisting of all tuples having a zero in position $q$. Under this identification, $C_{q}$ is a completely irreducible ideal of $R$, and $0=\bigcap_{q} C_{q}$ is an irredundant intersection. (In this example, $\operatorname{Soc} R$ is an essential submodule of $R$.) 
In the preceding example, we could have equally well used several subrings (containing $\bigoplus_{p} F_{p}$ ) of $R$, e.g. the von Neumann regular ring over which all von Neumann regular rings are unital algebras; see Fuchs-Halperin [13].

Another criterion for the existence of an irredundant intersection representation of an ideal $A$ with completely irreducible ideals can be given in terms of the injective hull of $R / A$.

Theorem 2.7. The proper ideal $A$ of the ring $R$ admits an irredundant representation as an intersection of completely irreducible ideals $C_{i}(i \in I)$ if and only if the injective hull $E(R / A)$ of $R / A$ is an interdirect product of injective hulls of simple $R$-modules.

Proof. The 'only if' part of the theorem is an immediate consequence of Theorem 2.5. since the components $R / C_{i}$ in the subdirect product are $R$-modules each with a simple essential submodule.

Conversely, assume $E(R / A)$ is an interdirect product of injective hulls $E\left(S_{i}\right)$ of simple $R$-modules $S_{i}$. Define $R_{i}=\pi_{i}(R / A)$, where $\pi_{i}$ denotes the $i$ th coordinate projection of the product of the $E\left(S_{i}\right)$. Then $R / A$ is evidently a subdirect product of the $R_{i}$, and the $R_{i}$ are subdirectly irreducible. For each $i$, the submodule $E\left(S_{i}\right)$ of $E(R / A)$ has nonzero intersection with $R / A$, and this intersection is contained in the kernel of $\pi_{j}$ for each $j \neq i$. This shows that the representation is irredundant. By Theorem 2.5. $A$ admits an irredundant representation as an intersection of completely irreducible ideals.

For a proper ideal $A$ of $R$, let $S(A)$ denote the set of elements $r \in R$ that are not prime to $A$, i.e. that satisfy $A \subset A: r$.

Proposition 2.8. Let $A$ be a proper ideal of a ring $R$. If $A$ is an irredundant intersection of completely irreducible ideals $C_{i}$, then $S(A)$ is the union of maximal ideals of $R$, each of which is a strong Bourbaki associated prime of $A$.

Proof. Given $r \in R$, we have $r \in S(A) \Longleftrightarrow A \subset A: r$. Since $A: r=\bigcap_{i \in I}\left(C_{i}: r\right)$, we have, by Lemma 2.1(iii), $r \in S(A) \Longleftrightarrow C_{i} \subset C_{i}: r$ for some $i$. Letting $M_{i}=\left\{t \in R \mid C_{i} \subset C_{i}: t\right\}$, the adjoint prime of $C_{i}$, we see that $S(A)=\bigcup_{i \in I} M_{i}$.

The elements $u_{i} \in R$ introduced in Lemma 2.1 satisfy $M_{i}=C_{i}: u_{i}$ and $u_{i} \in C_{j}$ for $j \neq i$. Thus $A: u_{i}=M_{i}$, showing that $M_{i}$ is a strong Bourbaki associated prime of the ideal $A$.

We can now prove:

Corollary 2.9. Let $A=\bigcap_{i \in I} C_{i}$ be an irredundant intersection, where the $C_{i}$ are completely irreducible ideals. Then $A$ is an $M$-primal ideal if and only if all the $C_{i}$ are $M$-primal.

Proof. If all the completely irreducible ideals $C_{i}$ are $M$-primal, then by Proposition $2.8 S(A)=M$, so $A$ is $M$-primal. On the other hand, if $A$ is $M$-primal, i.e. if $S(A)=M$, then again by Proposition 2.8, all the adjoint primes of the $C_{i}$ must be equal to $M$.

This leads to a coarser intersection decomposition of ideals admitting irredundant intersections with completely irreducible ideals. 
Corollary 2.10. Assume that the proper ideal $A$ of $R$ has an irredundant representation as an intersection of completely irreducible ideals. Then $A$ is an irredundant intersection of primal ideals with distinct adjoint maximal ideals such that each primal component is an irredundant intersection of completely irreducible ideals.

Proof. Let $A=\bigcap_{i \in I} C_{i}$ be an irredundant intersection, where the $C_{i}$ are completely irreducible ideals. For each maximal ideal $M$ that is the adjoint prime of some $C_{i}$, let $A^{[M]}$ denote the intersection of the $C_{i}$ that are $M$-primal. By Corollary 2.9. $A^{[M]}$ is $M$-primal. Thus $A=\bigcap_{M \in \operatorname{Max} R} A^{[M]}$ is a decomposition of $A$ into an irredundant intersection of primal ideals with distinct adjoint primes $M$, and each $A^{[M]}$ is an irredundant intersection of completely irreducible ideals.

Since the isolated $M$-component $A_{(M)}$ of an ideal $A$ is the intersection of all $M$-primal overideals of $A$, it is clear that $A_{(M)} \subseteq A^{[M]}$ for each $M$.

It would be interesting to know if the converse of Corollary 2.10 is also true.

Example 2.11. It is possible that an ideal $A$ is an irredundant intersection of completely irreducible ideals $\left\{C_{i}\right\}_{i \in I}$ and also the intersection of relevant completely irreducible divisors $\left\{C_{i}^{\prime}\right\}_{i \in I^{\prime}}$, where no subset of $\left\{C_{i}^{\prime}\right\}_{i \in I^{\prime}}$ gives an irredundant representation of $A$. Let $R$ be the ring defined in Example 2.4, It has been proved there that its zero ideal admits a representation as an irredundant intersection of completely irreducible ideals. Define $A_{n}(n>0)$ to be an ideal containing all $r_{i}(i<\omega, i \neq n)$, containing $e$, and maximal with respect to not containing $r_{n}$. Furthermore, for each $n<\omega$ let $B_{n}(n<\omega)$ be an ideal that contains all $r_{i}$ with $i>0$, contains the element $r_{0}-p_{0}^{n} p_{1}^{n} \cdots p_{n}^{n} e$, and is maximal with respect to not containing $r_{0}$. In view of Proposition 1.9, all of $A_{n}, B_{n}$ are relevant completely irreducible divisors of $A$. Evidently, $A=\bigcap_{n>0} A_{n} \cap \bigcap_{n<\omega} B_{n}$. Here each of the $A_{n}$, but none of $B_{n}$, is relevant for the intersection. Indeed, infinitely many $B_{n}$ can be deleted from this intersection as long as infinitely many $B_{n}$ remain. In view of this, this intersection cannot be made irredundant by canceling components.

\section{The question OF UNIQUENESS}

In her seminal paper [27] E. Noether proved that in a Noetherian ring every proper ideal $A$ is the finite irredundant intersection of irreducible ideals, and this intersection has the following uniqueness properties: (1) the number of irreducible components is unique, (2) the components satisfy the Replacement Property as defined in Theorem 3.2, and (3) the components are primary ideals and their set of prime radicals (along with their multiplicities) is uniquely determined by $A$.

Our next goal is to prove, if possible, analogous results for ideals $A$ that admit a representation as an irredundant intersection of completely irreducible ideals. Of course, the role of primary ideals will be taken over by primal ideals, and the set of prime radicals will be replaced by the set of adjoint maximal ideals.

In our attempt to generalize the Noetherian situation the first problem we face is that the cardinality of the set of completely irreducible components in an irredundant intersection need not be unique. This is illustrated by the following example.

Example 3.1. Let $\kappa$ be an infinite cardinal and let $\lambda$ be any cardinal such that $\kappa<\lambda \leq 2^{\kappa}$. There exists a ring $R$ that contains an ideal which is the irredundant intersection of $\kappa$ completely irreducible ideals and also the irredundant intersection 
of $\lambda$ completely irreducible ideals. Fix a prime integer $p$ and let $P=\prod_{\alpha<\kappa} Z_{\alpha}$ be the direct product of of $\kappa$ cyclic groups $Z_{\alpha}$ of order $p$. Give $P$ the trivial multiplication and make $R=\mathbb{Z} \oplus P$ into a ring as in Example 2.3. Evidently $P=\operatorname{Soc} R$ and $|P|=2^{\kappa}$. For $\beta<\kappa$, the product $P_{\beta}=\prod_{\alpha<\kappa, \alpha \neq \beta} Z_{\alpha}$ may be viewed as an ideal in $R$; it is completely irreducible, since it is maximal with respect to intersecting $Z_{\beta}$ in zero. Clearly, $0=\bigcap_{\beta<\kappa} P_{\beta}$ is an irredundant intersection with $\kappa$ components. Pick a subgroup $P^{\prime}$ of $P$ that contains the direct sum $\bigoplus_{\alpha<\kappa} Z_{\alpha}$ and has index $\lambda$ in $P$. Now $P=P^{\prime} \oplus \bigoplus_{\gamma<\lambda} W_{\gamma}$ for cyclic groups $W_{\gamma}$ of order $p$. Consider completely irreducible ideals $C_{\alpha}$ that contain $\bigoplus_{\gamma<\lambda} W_{\gamma}$ and are maximal with respect to intersecting $Z_{\alpha}$ in zero, and consider as well completely irreducible ideals $C_{\gamma}^{\prime}$ that contain $P^{\prime}$ and are maximal with respect to intersecting $W_{\gamma}$ in zero. Then the intersection $0=\bigcap_{\alpha<\kappa} C_{\alpha} \cap \bigcap_{\gamma<\lambda} C_{\gamma}^{\prime}$ is irredundant with $\lambda$ components.

As far as uniqueness of irredundant decompositions is concerned, we have the following:

Theorem 3.2. Let

$$
A=\bigcap_{i \in I} C_{i}=\bigcap_{j \in J} B_{j}
$$

be two irredundant intersection representations of the proper ideal $A$ of the ring $R$ with completely irreducible ideals $C_{i}, B_{j}$. Then:

(i) Each $M \in \operatorname{Max} R$ that occurs as the adjoint prime of some primal ideal $C_{i}$ also occurs as the adjoint prime of some $B_{j}$. If it occurs a finite number of times in one intersection, then it occurs the same number of times in the other intersection.

(ii) The intersections have the Replacement Property: for each $C_{k}$ there is a $B_{j}$ such that replacing $C_{k}$ by $B_{j}$ in the first intersection, we obtain an irredundant representation of $A$.

Proof. We start the proof with (ii). (Note that in the first paragraph of its proof irredundancy is irrelevant.)

(ii) Write $\bar{C}_{k}=\bigcap_{i \neq k} C_{i}$ and $A_{k j}=\bar{C}_{k} \cap B_{j}$. The $R$-module $\left(C_{k}+\bar{C}_{k}\right) / C_{k} \cong$ $\bar{C}_{k} /\left(C_{k} \cap \bar{C}_{k}\right) \cong \bar{C}_{k} / A$ has an essential simple socle. Therefore, $A=\bigcap_{j \in J} A_{k j}$ with $A_{k j} \subseteq \bar{C}_{k}$ implies that one of $A_{k j}(j \in J)$ must be equal to $A$, say, $\bar{C}_{k} \cap B_{j}=A$. Thus $C_{k}$ can be replaced by $B_{j}$.

To show that if $A=\bigcap_{i \in I} C_{i}$ is irredundant, then so is the new intersection, assume by way of contradiction that $C_{\ell}(\ell \neq k)$ can be omitted from the new intersection, i.e. $A=\bar{C}_{k} \cap \bar{C}_{\ell} \cap B_{j}$ (where $C_{k}$ and $C_{\ell}$ are missing). What has been proved is applied to this decomposition and to the original one in order to conclude that here $B_{j}$ can be replaced by one of the $C_{i}$. After we do this, at least one of $C_{k}$ and $C_{\ell}$ would be missing from the intersection, contradicting the irredundancy of the decomposition $A=\bigcap_{i \in I} C_{i}$.

(i) Both $C_{k}$ and its replacement $B_{j}$ satisfy $\bar{C}_{k} \cap C_{k}=A=\bar{C}_{k} \cap B_{j}$. Hence the covers satisfy $C_{k}^{*} / C_{k} \cong \operatorname{Soc}\left(\bar{C}_{k} / A\right) \cong B_{j}^{*} / B_{j}$. It follows that both $C_{k}$ and $B_{j}$ are $M$-primal for the maximal ideal $M$ that satisfies $\operatorname{Soc}\left(\bar{C}_{k} / A\right) \cong R / M$. Thus an $M$-primal completely irreducible ideal is always replaced by another $M$-primal ideal in an irredundant intersection.

Now if the first intersection contains $n$ completely irreducible ideals that are $M$-primal, then successively replacing them by (necessarily distinct) completely 
irreducible ideals from the second intersection, it follows that the second intersection contains at least $n M$-primal ideals. Hence the claim is evident.

Dilworth-Crawley [7, Theorem 4.2] prove an analogue of (ii) for compactly generated modular lattices under the hypothesis that the lattice is atomic (in our setting, this corresponds to the case where Soc $(R / A)$ is essential in $R / A)$. They also characterize atomic lattices where the components in irredundant intersections are unique. Since we do not have atomicity in general (see Example 2.4), in our search for uniqueness an independent approach is needed.

Remark 3.3. In [21] the rings are characterized for which every ideal can be represented uniquely as an irredundant intersection of completely irreducible ideals. These rings are necessarily arithmetical. Indeed, it is shown in 21 that if $\mathcal{A}$ is the set of ideals of a ring $R$ that are finite intersections of completely irreducible ideals, then $R$ is arithmetical if and only if for each $A \in \mathcal{A}$ the representation of $A$ as an irredundant intersection of completely irreducible ideals is unique.

\section{WHEN EVERY IDEAL IS AN IRREDUNDANT INTERSECTION}

In this section we characterize the rings in which every ideal is an irredundant intersection of completely irreducible ideals. We start with the following observation.

Lemma 4.1. Consider the following statements about a proper ideal $A$ of the ring $R$ :

(i) $A$ is an irredundant intersection of completely irreducible ideals.

(ii) $\operatorname{Soc}(R / A)$ is an essential submodule of $R / A$.

Then (ii) implies (i). On the other hand, if (i) holds for every proper ideal of R, then (ii) also holds for every proper ideal of $R$.

Proof. Assume that (ii) holds and let $E(R / A)$ denote the injective hull of $R / A$. Express $\operatorname{Soc}(R / A)$ as the direct sum $\bigoplus_{i \in I} S_{i}$ of simple $R$-modules $S_{i}$, and let $E\left(S_{i}\right)$ be a maximal essential extension of $S_{i}$ in $E(R / A)$. Then $E\left(S_{i}\right)$ is an injective hull of $S_{i}$ and $\bigoplus_{i \in I} E\left(S_{i}\right)$ is an essential submodule of $E(R / A)$. The canonical map of the direct sum $\bigoplus_{i \in I} E\left(S_{i}\right)$ into the direct product $\prod_{i \in I} E\left(S_{i}\right)$ extends to an injection of $E(R / A)$ into $\prod_{i \in I} E\left(S_{i}\right)$. Thus $E(R / A)$ is an interdirect sum of the $E\left(S_{i}\right)$. By Theorem 2.7. $A$ is an irredundant intersection of completely irreducible ideals.

Assume that (i) holds for every proper ideal of $R$ and let $A$ be a proper ideal of $R$. By Lemma 2.1, Soc $(R / A)$ cannot be trivial. Let $B / A$ be a maximal essential extension of Soc $(R / A)$ in $R / A$. Then $R / B$ has trivial socle, so $B$ cannot admit an irredundant representation as intersection of completely irreducible ideals. It follows that $B=R$. This means that $\operatorname{Soc}(R / A)$ is an essential submodule in $R / A$.

Recall that a ring $R$ is semi-artinian if every nonzero $R$-module contains a simple $R$-module. We will call a domain almost semi-artinian if every proper factor ring is semi-artinian.

Theorem 4.2. Every ideal of a ring is an irredundant intersection of completely irreducible ideals exactly if the ring is semi-artinian. Every nonzero ideal of an 
integral domain is an irredundant intersection of completely irreducible ideals if and only if the integral domain is almost semi-artinian.

Proof. Lemma 4.1 implies that all the ideals of a ring $R$ have the stated property if and only if $\operatorname{Soc}(R / A)$ is essential in $R / A$ for all proper ideals $A$ of $R$. This is the case exactly if $R$ is semi-artinian.

The claim concerning domains is an immediate consequence of the first part.

Example 4.3. A ring that is perfect in the sense of Bass 2] is semi-artinian. Perfect rings can be defined in several equivalent ways; for example, the ring $R$ is perfect is equivalent to each of the following statements: (i) every flat $R$-module is projective, (ii) $R$ satisfies the minimum condition for principal ideals (see e.g. Anderson-Fuller [1, p. 315]).

Example 4.4. Consider the Bézout domain $R$ of dimension 1 constructed by Heinzer-Ohm [20, Example 2.2]. For this ring $R, \operatorname{Spec} R$ is not Noetherian, but all of its localizations at maximal ideals are rank-one discrete valuation domains. Moreover, all but one of its maximal ideals are principal. It can be seen that this example is almost semi-artinian. Thus an almost semi-artinian domain need not have Noetherian spectrum.

More interesting examples are those almost semi-artinian domains that are also $h$-local, where an integral domain is $h$-local if each nonzero element is contained in only finitely many maximal ideals and each nonzero prime ideal is contained in a unique maximal ideal.

Example 4.5. The almost perfect domains introduced by Bazzoni-Salce [4 are defined as domains such that every proper factor ring is a perfect ring. They prove that an almost perfect domain can be characterized as an $h$-local domain $R$ such that for every nonzero proper ideal $A$, the factor ring $R / A$ contains a simple $R$-module. We are interested in obtaining more information about the ideal structure of almost perfect domains. An almost perfect domain is a Matlis domain of dimension $\leq 1$ with Noetherian prime spectra, where an integral domain $R$ is a Matlis domain if its field of fractions has projective dimension at most 1 as an $R$ module. The inclusion here is strict since a rank-one nondiscrete valuation domain is a Matlis domain of dimension 1 that fails to be almost perfect.

In an almost perfect domain, irreducible ideals are primary. Indeed, by [1] a necessary and sufficient condition for an irreducible ideal $A$ of a ring $R$ to be primary is that any strictly ascending chain of the form

$$
A \subset A: b_{1} \subset A: b_{1} b_{2} \subset \cdots \subset A: b_{1} b_{2} \cdots b_{n} \subset \cdots
$$

for any sequence $b_{1}, b_{2}, \ldots, b_{n}, \ldots$ in $R$ terminates. This is evidently true if the descending chain condition holds for principal ideals $\bmod A$.

Since an almost perfect domain $R$ is $h$-local, every nonzero ideal $A$ of $R$ is contained in at most a finite number of maximal ideals, $M_{1}, \ldots, M_{n}$. Consequently, we can write

$$
A=A_{\left(M_{1}\right)} \cap \cdots \cap A_{\left(M_{n}\right)} .
$$

The localization at a maximal ideal of a domain of dimension one is again of dimension one, and if $R$ is a one-dimensional quasilocal domain with maximal ideal $M$, then every nonzero proper ideal of $R$ is $M$-primary. Consequently, the isolated $M_{i}$-component $A_{\left(M_{i}\right)}$ must be $M_{i}$-primary for each $i=1, \ldots, n$. 
We record as Theorem 4.6 an immediate consequence of [5, Theorem 8].

Theorem 4.6. In an almost perfect domain $R$, every nonzero ideal $A$ is the product of pairwise comaximal primary ideals:

$$
A=B_{1} B_{2} \cdots B_{n},
$$

where the $B_{i}$ are primary ideals with distinct maximal ideals $M_{i}$ as radicals. This product representation is unique up to the order of the factors. Since an almost perfect domain is almost semi-artinian, the $B_{i}$ are irredundant intersections of completely irreducible $M_{i}$-primary ideals.

In particular, it follows that an almost perfect domain is Laskerian. We recall that a ring is said to be strongly Laskerian if it is Laskerian and every primary ideal contains a power of its radical. If $A$ is a nonzero ideal of a one-dimensional strongly Laskerian domain $R$, then $R / A$ satisfies the minimum condition for principal ideals, for $R / A \cong \prod_{i=1}^{n} R_{i}$, where each $R_{i}$ is a zero-dimensional ring with nilpotent maximal ideal $M_{i}$. If $r_{i}$ is a positive integer such that $M_{i}^{r_{i}}=0$, then a strictly descending chain of principal ideals in $R_{i}$ has length at most $r_{i}$. It follows that a strictly descending chain of principal ideals in $R / A$ has length at most $r_{1}+\cdots+r_{n}$. Thus $R$ is almost perfect.

Remark 4.7. The representations of the ideals $B_{i}$ of Theorem 4.6 as irredundant intersections of completely irreducible $M_{i}$-primary ideals may be infinite intersections. For example, if $R$ with maximal ideal $M$ is a one-dimensional local strongly Laskerian domain that is not Noetherian, then $M / M^{2}$ is infinite dimensional as a vector space over $R / M$. Since $R$ is almost semi-artinian, every nonzero ideal of $R$ is an irredundant intersection of completely irreducible ideals. However, every representation of $M^{2}$ as an intersection of completely irreducible ideals is an infinite intersection (see for example part (iv) of Lemma 2.1).

\section{Arithmetical Rings}

Proposition 1.4 and Corollary 1.5 characterize the completely irreducible ideals of a Noetherian ring. In this section we give an explicit description of the completely irreducible ideals in a much different setting, that of an arithmetical ring. These rings arise naturally in the consideration of irreducible ideals. In Theorem 1.8 and Remark 1.6 of [14 it is shown that the following are equivalent: (i) the ring $R$ is arithmetical, (ii) every primal ideal of $R$ is irreducible, (iii) the ideal $A_{(P)}$ is irreducible for every ideal $A$ and prime ideal $P$ of $R$ with $A \subseteq P$.

We first consider the special case of a valuation ring, where by a valuation ring we mean a ring in which the ideals are linearly ordered with respect to inclusion.

Lemma 5.1. Let $M$ be a maximal ideal of the ring $R$. The following statements are equivalent:

(i) $R$ is a valuation ring with maximal ideal $M$.

(ii) The set of proper completely irreducible ideals of $R$ is precisely the set of ideals of the form $r M$, where $r$ is a nonzero element of $R$.

(iii) For every nonzero $r \in R$, the ideal $r M$ is irreducible.

Proof. (i) $\Rightarrow$ (ii) Let $C$ be a completely irreducible ideal of $R$. Since $C^{*} / C$ is a simple $R$-module and $R$ is a valuation ring, $C^{*}$ must be a principal ideal of $R$ and $C=M C^{*}$. On the other hand, every ideal of the form $r M, r \in R$ and $r$ 
nonzero, is completely irreducible by Theorem 1.3(v) and the fact that every ideal of a valuation ring is irreducible.

(ii) $\Rightarrow$ (iii) This is clear.

(iii) $\Rightarrow$ (i) We first observe that (iii) implies $R$ is quasilocal. For if $r$ is not in $M$ but is in some other maximal ideal of $R$, then $r M=M \cap r R$ is an irredundant intersection, contradicting the fact that $r M$ is irreducible.

Let $x$ and $y$ be nonzero elements of $R$. We show that the ideals $x R$ and $y R$ are comparable. Notice that $M=x M: x$ and $M=y M: y$. The ideals $x M$ and $y M$ are irreducible, so by Theorem 1.3(v) $x M$ and $y M$ are completely irreducible. Thus it follows that $x R$ and $y R$ are the respective covers (unique minimal overideals) of $x M$ and $y M$. Let $I=(x, y) R$ and let $A$ be an ideal of $R$ with $M I=M x+M y \subset A$. Since $x R$ is the cover of $x M$ and $y R$ is the cover of $y M$, we must have $x \in A$ and $y \in A$. Also $M I \subset I$. Therefore $I$ is the unique minimal overideal of $M I$, and $M I$ is completely irreducible with cover $I$. In particular, $M I$ is irreducible, so $I / M I$ has dimension one as an $R / M$-vector space. Thus $I=x R+M I$ or $I=y R+M I$. Since $R$ is quasilocal, it follows that $I=x R$ or $I=y R$, proving that $x R$ and $y R$ are comparable.

Lemma 5.2. Let $A$ be an ideal of a ring $R$. If $M$ is a maximal ideal of $R$, then $M A_{(M)}=(M A)_{(M)}$.

Proof. Note first that $M A_{(M)} \subseteq(M A)_{(M)}$. For if $m \in M$ and $x \in A_{(M)}$, then there exists $y \in R \backslash M$ such that $x y \in A$, so that $m x y \in M A$ and $m x \in(M A)_{(M)}$. To complete the proof we verify locally that $(M A)_{(M)}=M A_{(M)}$. Clearly $(M A)_{(M)} R_{M}=$ $M A R_{M}=M A_{(M)} R_{M}$, so suppose $N$ is a maximal ideal of $R$ distinct from $M$. Then $M A_{(M)} \subseteq(M A)_{(M)}$ implies that $A_{(M)} R_{N}=M A_{(M)} R_{N} \subseteq(M A)_{(M)} R_{N} \subseteq$ $A_{(M)} R_{N}$; hence $M A_{(M)} R_{N}=(M A)_{(M)} R_{N}$.

Theorem 5.3. A ring $R$ is arithmetical if and only if the completely irreducible proper ideals of $R$ are precisely the ideals of the form $M B_{(M)}$, where $M$ is a maximal ideal and $B$ is a principal ideal having the property that $B R_{M} \neq 0$.

Proof. Suppose that $R$ is an arithmetical ring. Let $C$ be a completely irreducible proper ideal of $R$ and let $M$ be the adjoint (maximal) ideal of $C$. By Theorem 1.3 , $C R_{M}$ is completely irreducible, and by Lemma 5.1, $C R_{M}=(r / 1) M R_{M}$, where $r \in R$ is such that its image $r / 1$ in $R_{M}$ is nonzero. Define $B=r R$. Since for every ideal $A$ of $R$, the ideal $A_{(M)}$ is the preimage of $A R_{M}$ under the canonical mapping $R \rightarrow R_{M}$, and since $C=C_{(M)}$, we have $C=(M B)_{(M)}$. Therefore by Lemma $5.2 C=M B_{(M)}$, and every completely irreducible ideal of $R$ has the stated form. By Lemmas 5.1 and 5.2 and Theorem 1.3(vi), every ideal of the stated form is completely irreducible.

Conversely, assume that the completely irreducible proper ideals of $R$ are precisely the ideals of the form $M B_{(M)}$, where $M$ is a maximal ideal and $B$ is a principal ideal of $R$ having the property that $B R_{M} \neq 0$. It suffices to prove that $R_{M}$ is a valuation ring for every maximal ideal $M$ of $R$. By Lemma 5.1 it suffices to show that $(r / 1) M R_{M}$ is irreducible for all elements $r \in R$ such that $r / 1 \in R_{M}$ is nonzero. By assumption, $M(r R)_{(M)}$ is an irreducible ideal of $R$, and this implies that $(r / 1) M R_{M}$ is an irreducible ideal of $R_{M}$ (Remark 1.6 of [14]).

An ideal $A$ of a domain $R$ is said to be archimedean if its only endomorphisms are multiplications by elements of $R$, i.e. if its endomorphism $\operatorname{ring}, \operatorname{End}(A)$, is equal 
to $R$ [18, page 71]. We observe in Remark 5.4 that completely irreducible ideals of a Prüfer domain need not be archimedean.

Remark 5.4. Let $R=\mathbb{Z}+x \mathbb{Q}[[x]]$ be the ring of power series in $x$ with rational coefficients whose constant term is an integer. It is known that $R$ is a Prüfer domain, $\operatorname{dim} R=2$ and $P=x \mathbb{Q}[[x]]$ is the unique nonmaximal nonzero prime ideal of $R$. Moreover $P$ is contained in each maximal ideal of $R$, and for $p \in \mathbb{Z}$ a prime integer, $p R$ is a maximal ideal of $R$. Let $V$ denote the valuation overring $R_{2 R}=\mathbb{Z}_{2 \mathbb{Z}}+P$, and consider the principal ideal $A=x V$ of $V$. Since $x \in P, A$ is also an ideal of $R$ and is completely irreducible as an ideal of $R$ with adjoint maximal ideal $2 R$ and cover $(x / 2) V$. Observe that $\operatorname{End}(A)$ is the proper valuation overring $V$ of $R$. Therefore $A$ is not archimedean as an ideal of $R$. Since $A$ is completely irreducible, it is not possible to represent $A$ as an intersection of archimedean ideals of $R$.

For a prime ideal $P$ of a Prüfer domain, Fuchs and Mosteig prove in 17, Lemma $4.3]$ that the $P$-primal ideals form a semigroup under ideal multiplication. We generalize this result in Theorem 5.6. In the proof of Theorem 5.6. we use the following lemma.

Lemma 5.5. Let $M$ be a maximal ideal of an arithmetical ring $R$. If $A$ is a finitely generated ideal of $R$ contained in $M$ such that $A R_{M} \neq 0$, then $A_{(M)}$ is an $M$-primal ideal of $R$.

Proof. Observe that $A_{(M)}$ is an $M$-primal ideal of $R$ if and only if $M$ is the unique maximal Krull associated prime of $A_{(M)}$. If $P$ is a Krull associated prime of $A_{(M)}$, then necessarily $P \subseteq M$, so to show that $A_{(M)}$ is an $M$-primal ideal it suffices to show that $M$ is a Krull associated prime of $A_{(M)}$. Now by Lemma 2.4 of [14, $M$ is a Krull associated prime of $A_{(M)}$ if and only if $M R_{M}$ is a Krull associated prime of $A R_{M}$. Also, a consequence of Lemma 1.5 in [15] is that every nonzero principal ideal of a valuation ring $V$ has adjoint prime the maximal ideal of $V$. Thus $A R_{M}$ is $M R_{M}$-primal, and it follows that $A_{(M)}$ is an $M$-primal ideal of $R$.

Theorem 5.6. Let $P$ be a prime ideal of an arithmetical ring $R$. If $A$ and $B$ are $P$-primal ideals such that for all maximal ideals $M$ of $R, A B R_{M}$ is a nonzero ideal of $R_{M}$, then $A B$ is a P-primal ideal of $R$. In particular the set of regular P-primal ideals of $R$ is a semigroup under ideal multiplication.

Proof. We first make two observations:

(a) If $C$ is an ideal of $R$, then $P$ is a Krull associated prime of $C$ if and only if $P R_{M}$ is a Krull associated prime of $C R_{M}$ for some (equivalently, all) maximal ideal(s) $M$ containing $P$. This is proved in Lemma 2.4 of [14].

(b) If $C$ is a primal ideal of $R$, then $P$ is the adjoint prime of $C$ if and only if for each maximal ideal $M$ of $R$ containing $P, C R_{M}$ is a $P R_{M}$-primal ideal of $R_{M}$. This is an application of (a) and the fact that an ideal $C$ is $P$-primal if and only if $P$ is the unique maximal member of the set of Krull associated primes of $A$.

We now prove the theorem. Suppose first that $R$ is a valuation ring. Then $A B$ is irreducible, hence primal, with adjoint prime, say $Q$. We claim that $Q \subseteq P$. If this is not the case, then $P \subset Q$, and since $A B$ is $Q$-primal, there exists $q \in Q \backslash P$ such that $q x \in A B$ for some $x \in Q \backslash A B$. Since $B$ is $P$-primal, $B: q=B$. Also, $B \subseteq q R$, so $B=q C$ for some ideal $C$. Necessarily $B \subseteq C \subseteq B: q=B$, so this forces $B=C$. Thus $q B=B$ and $q A B=A B$. Since $R$ is a valuation ring and $x \notin A B$, we have $A B \subseteq x R$. Thus $x q R \subseteq A B=q A B \subseteq x q R$, and we conclude 
that $x q R=A B$. It follows that $x q^{2} R=q A B=A B=x q R$, so that $x q=r x q^{2}$ for some $r \in R$. But this implies that $(1-r q) x q=0$, and since $1-r q$ is a unit in $R$, $x q=0$, contradicting the assumption that $x q R=A B \neq 0$. Thus $Q \subseteq P$.

We show that in fact $Q=P$. If this is not the case, then $Q \subset P$ and there exists $p \in P \backslash Q$. Since $A B$ is $Q$-primal, $A B: p^{2}=A B$. Since $A$ and $B$ are $P$-primal, there exist $x \in P \backslash A$ and $y \in P \backslash B$ such that $x p \in A$ and $y p \in B$. Hence $x y \in A B: p^{2}=A B$. Since $R$ is a valuation ring, $A \subset x R$ and $B \subset y R$. We conclude that $x y R \subseteq A B \subseteq x y R$. Thus by Lemma 5.5 and the fact that $A B \neq 0$, we have that $A B$ is an $M$-primal ideal of $R$. This forces $Q=P=M$, a contradiction to the assumption that $Q \subset P$. Hence $P=Q$ and $A B$ is $P$-primal.

We now consider the general case in which $R$ is not necessarily a valuation ring. As noted above in (b), to prove that $A B$ is $P$-primal, it suffices to show that $P$ is the unique maximal member of the set of Krull associated primes of $A B$. By (b) $A R_{M}$ and $B R_{M}$ are $P R_{M}$-primal ideals of $R$, and since $R_{M}$ is a valuation ring we have by the case considered above that $A B R_{M}$ is a $P R_{M}$-primal ideal. By (a) $P$ is a Krull associated prime of $A B$. To complete the proof it remains to show that $P$ is the unique maximal member of the set of Krull associated primes of $A B$. Suppose that $Q$ is a Krull associated prime of $A B$, and let $M$ be a maximal ideal of $R$ containing $Q$. By (a) $Q R_{M}$ is a Krull associated prime of $A B R_{M}$. Since $A B R_{M}$ is $P R_{M}$-primal, $Q R_{M} \subseteq P R_{M}$, so that $Q \subseteq P$. This completes the proof that every Krull associated prime of $A B$ is contained in $P$.

Remark 5.7. Simple examples show that even in a valuation ring $R$ with maximal ideal $M$ it is possible to have a proper ideal $A$ that is completely irreducible (and therefore $M$-primal) such that $A^{2}$ is not $M$-primal. Let $V$ be a valuation domain whose value group is $\Gamma=\mathbb{Z} \oplus \mathbb{Z}$ with lexicographic ordering. Then the maximal ideal of $V$ is principal generated by an element $x$, where the value $v(x)=(0,1)$ is the smallest positive element in $\Gamma$. Let $y \in V$ have value $(1,0)$, and let $Q$ be the prime ideal of $V$ of height one. Thus $Q$ is generated by elements $z$ having value $v(z)=(1, b)$, where $b \in \mathbb{Z}$ may be negative. Notice that $Q^{2} \subset y V \subset Q$ and that $Q^{2}$ is $Q$-primary. Define $R=V / Q^{2}$. Then $R$ is a valuation ring with maximal ideal $M=x R$. The ideal $A=y R$ is completely irreducible, so $M$-primal. However, $A^{2}=(0)$ is not $M$-primal. For a specific realization of this example, let $x, y$ be indeterminates over a field $k$, let $W=k(x)[y]_{(y)}$, let $Q=y W$, and set $V=k[x]_{(x)}+Q$. Then $V$ is a rank-two valuation domain with principal maximal ideal $x V$ and height-one prime $Q$. The valuation $\operatorname{ring} R=V / Q^{2}$ has maximal ideal $M=x R$. Since $Q^{2}$ is $Q$-primary, the ideal (0) of $R$ is not $M$-primal. The principal ideal $A=y R$ is a completely irreducible ideal and therefore is $M$-primal. However, $A^{2}=(0)$ is not $M$-primal.

If we consider only those completely irreducible regular ideals whose adjoint prime is a fixed maximal ideal $M$, then we can verify:

Theorem 5.8. Let $R$ be an arithmetical ring and let $M$ be a maximal ideal of $R$. The set $\mathcal{F}$ of completely irreducible regular ideals of $R$ with adjoint maximal ideal $M$ is closed under ideal-theoretic multiplication, and $\mathcal{F}$ with this multiplication is a totally ordered cancellative semigroup.

Proof. We show first that: $(\star)$ If $I$ and $J$ are principal ideals of $R$ and $I_{(M)}$ and $J_{(M)}$ are regular ideals of $R$, then $(I J)_{(M)}=I_{(M)} J_{(M)}$. Suppose that $I$ or $J$, say $I$, is not contained in $M$. Then $I J R_{M}=J R_{M}$, so that $(I J)_{(M)}=J_{(M)}$, and since 
$I \nsubseteq M, I_{(M)}=R$. Thus if $I$ or $J$ is not contained in $M$, then $(\star)$ holds. Otherwise, both $I$ and $J$ are contained in $M$, and by Lemma 5.5 $I_{(M)}$ and $J_{(M)}$ are $M$-primal ideals of $R$. As a product of regular $M$-primal ideals, $I_{(M)} J_{(M)}$ is $M$-primal by Theorem 5.6. Thus $\left(I_{(M)} J_{(M)}\right)_{(M)}=I_{(M)} J_{(M)}$, so since $I J \subseteq I_{(M)} J_{(M)}$ we have that $(I J)_{(M)} \subseteq I_{(M)} J_{(M)}$, and it remains to verify the reverse inclusion. Suppose that $x \in I: b$ and $y \in J: c$ for some $b, c \in R \backslash M$. Then $x y \in I J: b c \subseteq(I J)_{(M)}$ since $b c \notin M$. It then follows that $I_{(M)} J_{(M)} \subseteq(I J)_{(M)}$, and this proves $(\star)$.

Now let $A$ and $B$ be in $\mathcal{F}$. Then $A B$ is an $M$-primal ideal by Theorem 5.6, By Theorem 5.3. $A=M J_{(M)}$ and $B=M K_{(M)}$ for some principal ideals $J$ and $K$ of $R$. Since $A$ and $B$ are regular ideals, so are $J_{(M)}$ and $K_{(M)}$. Now if $M \neq M^{2}$, then since by Theorem 5.6, $M^{2}$ is primal, hence irreducible, it follows that $M / M^{2}$ is a onedimensional $R / M$-vector space. Hence if $M \neq M^{2}, M=I_{(M)}$ for some principal ideal $I \subseteq M$. In this case we have by $(\star)$ (and the fact that $M$ is regular) that $A B=I_{(M)} J_{(M)} K_{(M)} M=(I J K)_{(M)} M$, so $A B \in \mathcal{F}$ by Theorem 5.3. Otherwise, if $M=M^{2}$, then again by $(\star) A B=(I J)_{(M)} M$ and by Theorem 5.3, $A B \in \mathcal{F}$.

Now assume $A C=B C$, where $A, B, C$ are in $\mathcal{F}$. Using Theorem 5.3, write $A=(M J)_{(M)}, B=(M K)_{(M)}$ and $C=(M L)_{(M)}$ for principal ideals $J, K$, and $L$ of $R$. Then $A C R_{M}=B C R_{M}$ implies that $M^{2} J L R_{M}=M^{2} K L R_{M}$. Moreover $L R_{M}$ is a regular principal ideal of $R_{M}$ since $C$ is regular and $C \subseteq L_{(M)}$ implies $C R_{M} \subseteq L R_{M}$. Thus $L R_{M}$ is a cancellation ideal of $R_{M}$ and $J M^{2} R_{M}=K M^{2} R_{M}$. If $M R_{M}=M^{2} R_{M}$, then clearly $A R_{M}=C R_{M}$. Otherwise, if $M R_{M} \neq M^{2} R_{M}$, then the irreduciblity of $M^{2} R_{M}$ implies (as above) that $M R_{M}$ is principal and regular, in which case also $A R_{M}=C R_{M}$. Therefore $A=A_{(M)}=C_{(M)}=C$.

Since $R$ is an arithmetical ring, the ideals of $R_{M}$ are linearly ordered with respect to inclusion and thus so are their preimages under the canonical map $R \rightarrow R_{M}$. Therefore for ideals $A$ and $B$ of $R$, the ideals $A_{(M)}$ and $B_{(M)}$ of $R$ are comparable. It follows that the ideals in $\mathcal{F}$ are totally ordered under inclusion.

\section{ACKNOWLEDGEMENT}

We thank the referee for numerous helpful suggestions about this paper.

\section{REFERENCES}

[1] F.W. Anderson and K.R. Fuller, Rings and Categories of Modules, Springer, 1974. MR0417223 (54:5281)

[2] H. Bass, Finitistic dimension and a homological generalization of semiprimary rings, Trans. Amer. Math. Soc. 95 (1960), 466-488. MR0157984 (28:1212)

[3] S. Bazzoni, Divisorial domains, Forum Math. 12 (2000), 397-419. MR1763897 (2001i:13031)

[4] S. Bazzoni and L. Salce, Almost perfect domains, Coll. Math. 95 (2003), 285-301. MR1968387 (2004b:13024)

[5] J. Brewer and W. Heinzer, On decomposing ideals into products of comaximal ideals, Comm. Algebra 30 (2002), 5999-6010. MR1941938 (2003h:13001)

[6] R.P. Dilworth, Abstract commutative ideal theory, Pacific J. Math. 12 (1962), 481-498. MR0143781 (26:1333)

[7] R.P. Dilworth and P. Crawley, Decomposition theory for lattices without chain conditions, Trans. Amer. Math. Soc. 96 (1960), 1-22. MR0118690(22:9461)

[8] M. Fontana and E. Houston, On integral domains whose overrings are Kaplansky ideal transforms, J. Pure Appl. Algebra 163 (2001), 173-192. MR1846660 (2002g:13003)

[9] M. Fontana, J.A. Huckaba, I.J. Papick and M. Roitman, Prüfer domains and endomorphism rings of their ideals, J. Algebra 157 (1993), 489-516. MR1220780 (94f:13011)

[10] M. Fontana and S. Kabbaj, Essential domains and two conjectures in ideal theory, Proc. Amer. Math. Soc. 132 (2004), 2529-2535. MR2054776 (2005c:13009) 
[11] L. Fuchs, A condition under which an irreducible ideal is primary, Quart. J. Math. Oxford 19 (1948), 235-237. MR0027266 (10:280c)

[12] L. Fuchs, On primal ideals, Proc. Amer. Math. Soc. 1 (1950), 1-6. MR0032584 (11:310d)

[13] L. Fuchs and I. Halperin, On the imbedding of a regular ring in a regular ring with identity, Fund. Math. 54 (1964), 285-290. MR0166211 (29:3488)

[14] L. Fuchs, W. Heinzer and B. Olberding, Commutative ideal theory without finiteness conditions: primal ideals, Trans. Amer. Math. Soc. 357 (2005), 2771-2798. MR2139527 (2006b:13006)

[15] L. Fuchs, W. Heinzer and B. Olberding, Maximal prime divisors in arithmetical rings, in Rings, Modules, Algebras, and Abelian Groups, Proceedings of the Algebra Conference Venezia, Marcel Dekker vol. 236 pages 189-203. MR2050712

[16] L. Fuchs, W. Heinzer and B. Olberding, Commutative ideal theory without finiteness conditions: irreducibility in the quotient field, in preparation.

[17] L. Fuchs and E. Mosteig, Ideal theory in Prüfer domains - an unconventional approach, J. Algebra 252 (2002), 411-430. MR1925145 (2003e:13028)

[18] L. Fuchs and L. Salce, Modules over non-Noetherian Domains, Math. Surveys 84 (2001). MR1794715 (2001i:13002)

[19] S. Gabelli and E. Houston, Ideal theory in pullbacks, Non-Noetherian commutative ring theory, 199-227, Math. Appl. 520, Kluwer Acad. Publ. Dordrecht 2000. MR1858163 (2003a:13001)

[20] W. Heinzer and J. Ohm, Locally Noetherian commutative rings, Trans. Amer. Math. Soc. 158 (1971), 273-284. MR0280472 (43:6192)

[21] W. Heinzer and B. Olberding, Unique irredundant intersections of completely irreducible ideals, J. Algebra 287 (2005), no. 2, 432-448. MR2134153 (2006a:13004)

[22] J. Iroz and D. Rush, Associated prime ideals in non-Noetherian rings, Can. J. Math. 36 (1984), 344-360. MR0749988 (85j:13002)

[23] W. Krull, Idealtheorie in Ringen ohne Endlichkeitsbedingung, Math. Ann. 101 (1929), 729744.

[24] W. Krull, Idealtheorie, Ergebnisse d. Math. (Berlin, 1935). MR0229623 (37:5197)

[25] K. A. Loper and M. Roitman, The content of a Gaussian polynomial is invertible, Proc. Amer. Math. Soc. 133 (2005), no. 5, 1267-1271. MR2111931 (2005j:13009)

[26] T. Lucas, Gaussian polynomials and invertibility, Proc. Amer. Math. Soc. 133 (2005), no. 7, 1881-1886. MR 2137851(2006a:13016)

[27] E. Noether, Idealtheorie in Ringbereichen, Math. Ann. 83 (1921), 24-66.

Department of Mathematics, Tulane University, New Orleans, Louisiana 70118

E-mail address: fuchs@tulane.edu

Department of Mathematics, Purdue University, West Lafayette, Indiana 47907

E-mail address: heinzer@math.purdue.edu

Department of Mathematical Sciences, New Mexico State University, Las Cruces, New Mexico 88003-8001

E-mail address: olberdin@emmy.nmsu.edu 